\title{
Endoscopic Gluteus Medius Repair Replicates Open, Knotless Repair With Similar Cyclic Loading Properties: A Cadaveric Study
}

\author{
Megan E. Flynn, M.D., David P. Beason, M.S., Katherine C. Bartush, M.D., \\ Michael K. Ryan, M.D., and Benton A. Emblom, M.D.
}

\begin{abstract}
Purpose: To compare the repair strength, gap formation, and mode of failure between endoscopic and open double-row gluteus medius repairs in a cadaveric model. Methods: Six pairs of fresh-frozen human cadavers were used in this study. Gluteus medius tears were created in an open fashion and then repaired with either open or endoscopic techniques. Specimens were manually preloaded to $5 \mathrm{~N}$, then cycled between 20-50 N for 150 cycles s. Then, a ramp to/s. Specimens were then returned to $10 \mathrm{~N}$ and ramped to failure at $1 \mathrm{~mm} / \mathrm{s}$. Gap formation and strengths of the construct were compared for the 2 techniques. Results: Biomechanical testing resulted in no significant differences in ultimate load $(P=.86)$ or gap formation $(P>.10)$ between groups. Ninety-two percent of specimens failed near the muscle origin on the ilium. Conclusions: This study shows that both open and endoscopic gluteus medius repairs are stronger than the muscle-bone interface in a cadaveric model and loaded biomechanically in tension between the ilium origin and femoral insertion. Further, endoscopic technique is able to replicate open, knotless gluteus medius repair technique in terms of gap formation in physiologic (i.e., subfailure) cyclic loading. Clinical Relevance: Gluteus medius tendinopathy is an increasingly common recognized etiology of lateral hip pain. When tears occur, debate exists over whether open or endoscopic repair procedures are optimal. Double-row endoscopic gluteus medius repair with knotless suture anchors may be an alternative to open repair.
\end{abstract}

G luteus medius tendinopathy has become an increasingly common recognized source of lateral hip pain. The gluteus medius is a broad lateral-based muscle of the hip that arises from the iliac crest and inserts on the lateral aspect of the greater trochanter. Tears of the gluteus medius tendon typically occur in women in their fourth through sixth decades of life. ${ }^{1}$ If conservative treatment fails, surgical treatment may become necessary.

Recent advancements in minimally invasive surgical techniques have led to a debate over whether open or endoscopic repair procedures are optimal. ${ }^{2}$ The debate between open versus endoscopic repair is similar to previous investigations involving rotator cuff repairs of
From the American Sports Medicine Institute (M.E.F., D.P.B., K.C.B., B.A.E.); and Andrews Sports Medicine $\theta$ Orthopaedic Center (M.K.R., B.A.E.), Birmingham, Alabama, U.S.A.

The authors report the following potential conflicts of interest or sources of funding: M.E.F. reports grants and nonfinancial support from Arthrex, during the conduct of the study; and nonfinancial support from Stryker Corporation and DePuy Synthes Sales, outside the submitted work. D.P.B. reports grants and nonfinancial support from Arthrex, during the conduct of the study. K.C.B. reports grants and nonfinancial support from Arthrex, during the conduct of the study; and nonfinancial support from Arthrex, Stryker Corp, Smith $\theta$ Nephew, OsteoCentric Technologies, Integra LifeSciences Corp, DePuy Synthes Sales, Gemini Mountain Medical, LLC, KCI USA, and Medtronic USA, outside the submitted work. M.K.R. reports grants and nonfinancial support from Arthrex, during the conduct of the study; and nonfinancial support from Linvatec Corp, Arthrex, DJO, Nutech Spine, Smith $\theta$ Nephew, ACUMED, PRIME SURGICAL, LLC, Stryker Corp, Zimmer Biomet Holdings, Avanos Medical, DePuy Synthes Sales, and ERMI, outside the submitted work. B.A.E. reports grants and nonfinancial support from Arthrex, during the conduct of the study; personal fees and nonfinancial support from Arthrex; and nonfinancial support from PRIME SURGICAL, Flexion Therapeutics, Zimmer Biomet Holdings, and Stryker Corp, outside the submitted work. This research was sponsored by Arthrex (AR1936000 to B.A.E.) in the form of direct funding, procurement of cadaveric specimens, and in-kind donation of surgical supplies. Full ICMJE author disclosure forms are available for this article online, as supplementary material.

Received February 23, 2021; accepted December 10, 2021.

Address correspondence to David P. Beason, M.S., 833 St. Vincent's Dr., Suite 205, Birmingham, AL 35205.E-mail:DavidB@asmi.org

(C) 2021 THE AUTHORS. Published by Elsevier Inc. on behalf of the Arthroscopy Association of North America. This is an open access article under the CC BY-NC-ND license (http://creativecommons.org/licenses/by-nc-nd/4.0/).

2666-061X/21249

https://doi.org/10.1016/j.asmr.2021.12.002 
the shoulder ${ }^{3}$; however, unlike the shoulder, the hip is necessary for ambulation and thus more difficult to protect during recovery. Therefore, surgical approach, technique, and ultimately repair strength are of utmost importance. ${ }^{2}$ Although both repair techniques aim to reattach the gluteus medius to its insertion site on the greater trochanter, it remains unclear which technique results in the biomechanically strongest construct. Studies have found patient-reported outcomes, pain reduction, and abduction power to be similar between endoscopic and open techniques. ${ }^{4}$

Despite the clinical similarities in prior reports, the authors believe there are several advantages to endoscopic repair. First, endoscopic repair allows for smaller incisions thereby decreasing infection rates, wound dehiscence, hematoma formation, and incisional pain. ${ }^{4}$ Endoscopy also allows for concomitant pathology to be addressed, if present. ${ }^{5}$ In chronic gluteus medius tears, endoscopy, like open techniques, also allows surgeons to mobilize retracted tendons and lyse scar tissue, with the added benefit of viewing other compartments of the hip without significant dissection. ${ }^{6}$ Patients with chondral changes not evident on preoperative imaging may be contraindicated for a gluteus medius repair procedure. $^{2}$ In cases of gluteus medius tears with concomitant degenerative changes, gluteus medius repair has not been found to improve pain, and only after hip arthroplasty is pain relief achieved. ${ }^{7}$

An association between open repair and increased retear rates may also exist. A systematic review by Chandrasekaran et al. ${ }^{4}$ compared open with endoscopic repair and found a combined retear rate of nearly $8 \%$ using open techniques compared with zero in endoscopic techniques. In light of the potential advantages over open surgical repair, endoscopic repair is appealing. There is a paucity of data elucidating the mode of failure in patients with gluteus medius repairs and, to the authors' knowledge, no study has quantified the strength of these repairs.

The purpose of this study is to compare the repair strength, gap formation, and mode of failure between endoscopic and open double-row gluteus medius repairs in a cadaveric model. Our hypothesis is that no difference exists in pull-out strength, gap formation, or rates, and modes of failure between endoscopic and open repair techniques.

\section{Methods}

Six fresh-frozen human cadaveric bilateral pelvic specimens (mean age $48 \pm 4$ years, age range 41-50 years) were used in this study. Selection criteria consisted of donors with an age less than 50 years at the time of death, body mass index less than 35, and without any previous history of hip injury, based on the medical social summary on the cadaver donor summary report. Each specimen contained one pelvis in its entirety. One side of each pelvis was selected for open gluteus medius repair whereas the contralateral side received the same repair endoscopically. Laterality was distributed evenly between the 2 groups. In this way, confounding variables including age, sex, bone quality, muscle bulk, and time from repair to biomechanical testing were reduced between comparison groups. The same repair technique was used for both open and arthroscopic repairs to standardize the repair itself. This technique is the preferred method of the senior author, and is outlined to follow. Each specimen was thawed to room temperature, which took approximately 72 hours, before repair. The gluteus minimus was identified and preserved.

\section{Repair Technique}

The cadavers were placed supine on a surgical table. For the open approach, a standard lateral approach to the hip was performed. The iliotibial band and tensor fascia lata muscle were incised in line with the skin incision to expose the gluteus medius and its insertion. First, a tear in the gluteus medius was created by incising it perpendicular to its fibers at the insertion and elevating it proximally with a 10 blade to create a fullthickness U-shaped tear (Fig 1). To begin the repair, 3 medial row anchors (3.9-mm knotless Corkscrew anchors; Arthrex, Naples, FL) were placed into the lateral facet of the greater trochanter at the site of the repair (Fig 2). These were loaded with \#2 coreless (Arthrex) suture, which was then passed in a horizontal mattress fashion through the musculotendinous junction. This was done by passing the repair suture from deep to superficial, and then back from superficial to deep. The repair suture was then pulled back into the knotless anchor, tensioning the horizontal mattress repair, and exiting back out through the superficial surface of the tendon. The knotless mechanism was deployed thereby reducing the tendon back to its footprint. For the lateral row, the 3 suture tails were loaded into a $4.75-\mathrm{mm}$

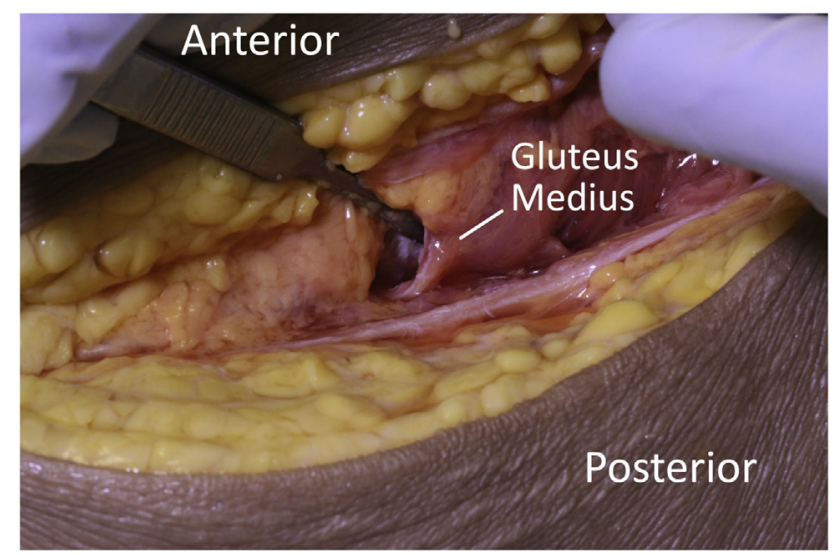

Fig 1. Creation of a U-shaped gluteus medius tear with a 10-blade scalpel from an open lateral approach. 


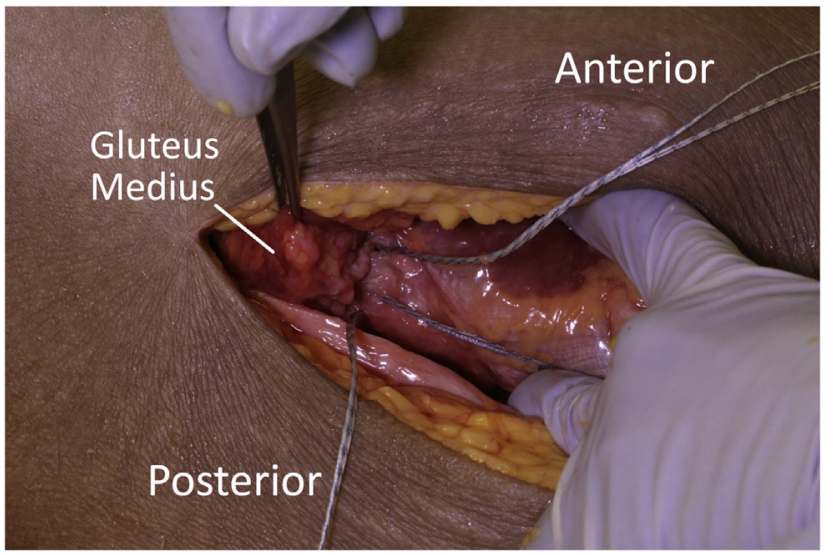

Fig 2. Three knotless suture anchors were placed in the proximal aspect of the tear.

PEEK SwiveLock anchor (Arthrex) and this was placed distally and laterally on the greater trochanter securing a "three and one" construct (Fig 3).

To create the same tear pattern for both endoscopic and open repairs, the same steps were repeated from incision to the creation of the U-shaped tear for the endoscopic repair specimens. Then, the skin was closed with a running \#2 VICRYL suture and anterolateral, mid-anterior, and distal anterolateral portals were established. A $30^{\circ}$ arthroscope was introduced into the peritrochanteric space via the use of the distal accessory anterolateral portal and anterolateral portals. The gluteal bursa was removed with a 4.2-mm hip-length Bone Cutter (Arthrex) shaver. The muscle belly of the vastus lateralis, gluteal sling, and proximal greater trochanter were identified in addition to the previouslymade gluteus medius tear. Then, using the standard distal anterolateral portal as the viewing portal, the same repair construct was employed as was used in the open procedure.

\section{Biomechanical Testing}

Following both open and endoscopic repair, all specimens were dissected such that the femur was disarticulated from the acetabulum and the gluteus medius was dissected down to its insertion on the ilium. Care was taken to preserve the entire muscle insertion on the ilium, keeping the gluteus medius intact from origin to repaired insertion, and all other tissue was removed, including excision of the gluteus minimis tendon from its insertion on the ilium.

The remaining femoral diaphysis was cemented in polymethyl methacrylate using modular acrylic tubing for placement in a servohydraulic mechanical test frame (MTS Systems, Eden Prairie, MN). The internal/ external rotation of the femur within the cylinder before cementing was adjusted so that the amount of tension on the repair (and on the insertion itself) would be distributed evenly over the course of the testing (i.e., the repair/insertion were oriented as horizontally as possible). This was placed into a custom jig rigidly attached to the base plate of the test frame, which oriented the femur at $30^{\circ}$ from horizontal with the proximal femur above the potted diaphysis (Fig 4). This was chosen based on trial testing as the orientation that would allow the gluteus medius tendon to have a naturally vertical line of action with the test frame actuator simulating abductor pull on the proximal femur. The ilium was loaded into a separate custom jig attached to the test frame actuator, which acted as a wedge to hold the ilium during biomechanical testing, but would not require clamping of the gluteus medius muscle-tendon complex (Fig 4). This configuration was chosen to preserve the gluteus medius anatomy from origin to insertion, thereby simulating a naturally occurring loading environment (aside from the isolation of the gluteus medius).

The entire gluteus medius complex was loaded in axial tension using the following protocol, which was modified from that of Dishkin-Paset et al. ${ }^{8}$ First, specimens were manually preloaded to $5 \mathrm{~N}$, followed by a 120 -second hold. Next, specimens were cycled in tension in force control between 20 and $50 \mathrm{~N}$ for 150 cycles s. Then, a ramp to/s. Then, a ramp to failure was performed in position control at $1 \mathrm{~mm} / \mathrm{s}$. In addition, 2-dimensional video was captured with a highdefinition camera (Canon USA, Inc., Melville, NY). The camera tracked the motion of prescribed surgical marker lines (Fig 5) created near the tendon-bone femoral insertion site as well as at 1 and $2 \mathrm{~cm}$ proximal to the insertion to aid in optical measurements of gap formation. Optical displacement calculations were later made by tracking the distance between the insertion site marker line and the $1-\mathrm{cm}$ marker line at the first cycle, tenth cycle, final cycle, and at the point of failure. Calibrated digital images were analyzed using ImageJ 1.53a (National Institutes of Health, Bethesda, $\mathrm{MD})$. Gap formation was calculated as the distance

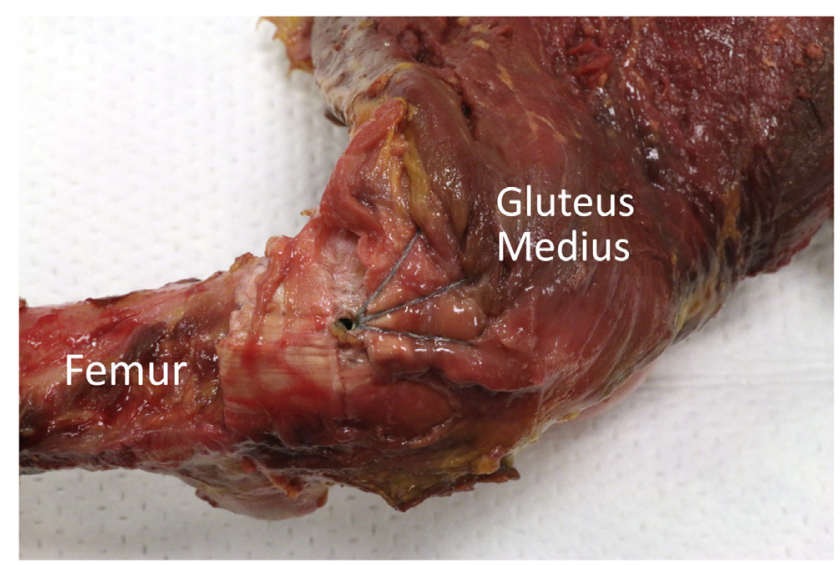

Fig 3. The "three-and-one" double-row construct is shown after dissection. 


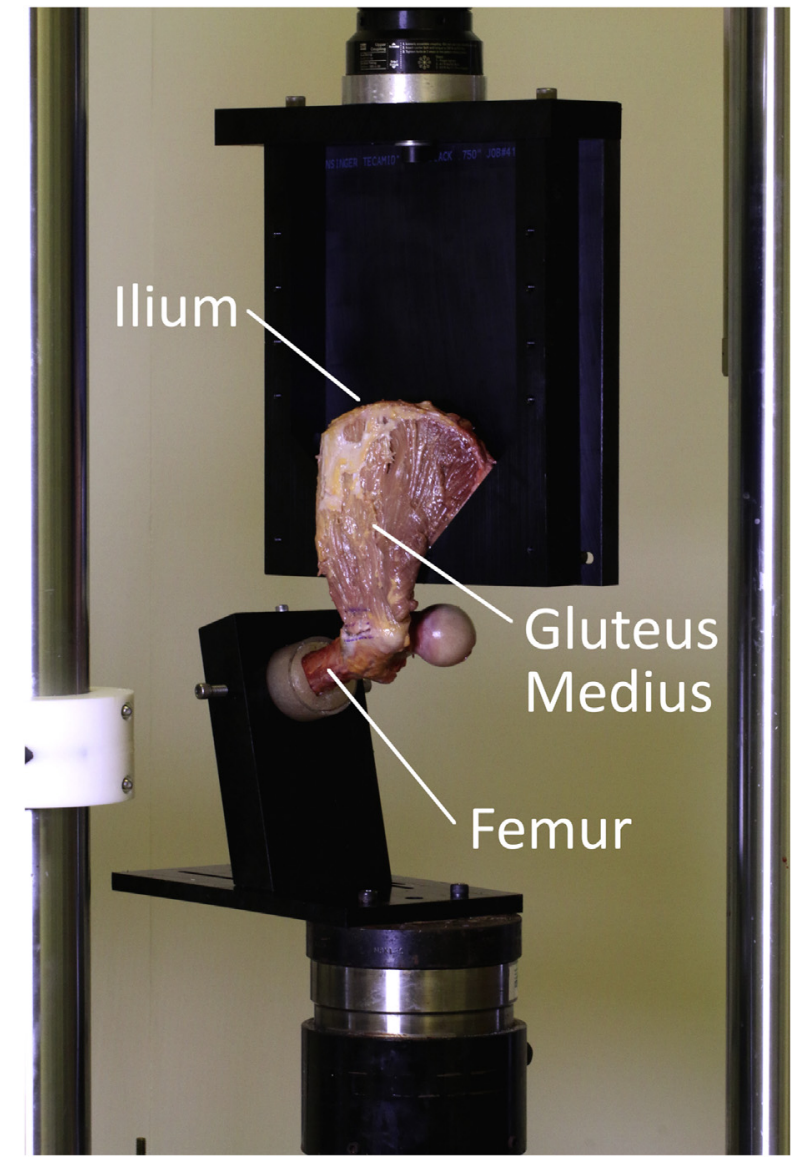

Fig 4. Overall setup of the servohydraulic test frame and custom jig.

between marker lines at the cycle of interest minus the initial distance at the end of the 120-second hold period. Measurements between open and endoscopic groups were compared via 2 -tailed paired $t$-tests, with statistical significance set at $P<.05$.

\section{Results}

Biomechanical testing resulted in no significant differences in ultimate load $(P=.86)$ or gap formation $(P>.10)$ between groups (Table 1). For both groups, a larger amount of repair gap formation occurred between the 1st and 10th cycles than between the 10th and 150th cycles (Fig 6). Failure tests resulted in 92\% (5/6 open, 6/6 endoscopic) of specimens failing near the muscle origin on the ilium. One endoscopic specimen failed in the muscle mid-substance. There were no repair site failures.

\section{Discussion}

In our study, biomechanical testing showed no differences in gap formation between open and endoscopic gluteus medius repairs when cyclically loaded according to our protocol. Our model was not able to quantify failure properties of the tears.
Advances in hip arthroscopic and endoscopic procedures have led to a substantial evolution in its use for a wide array of hip pathology. Unremitting hip pain and failure of conservative management with a presumed diagnosis of trochanteric bursitis constitute a relative indication for magnetic resonance imaging. ${ }^{9}$ Magnetic resonance imaging may reveal a previously unappreciated tear of the gluteus medius, which has been well described in the literature as a potential cause of greater trochanteric pain..$^{-11}$ In a matched-pair cohort study, Chandrasekaran et al. ${ }^{12}$ found patients who presented with abductor strength less than grade 4 and any deviation of gait pattern were more likely to be unresponsive to nonoperative management and require surgery.

In terms of surgical management, both open and endoscopic repairs have been shown to be effective in providing symptom improvement. In a large series of open abductor repairs, Walsh et al. ${ }^{13}$ reported substantial pain relief in 65 of 72 patients with an improvement in the Merle d'Aubigne and Postel hip score (maximum score 18 in a normal hip) from a preoperative mean of $10.85 \pm 0.30$ points (range $2-12$ points) to a mean of $16.65 \pm 0.35$ points (range 9-18 points) at 12 months' postoperatively. Improvement in gait also was found in these patients with $5 \%$ walking normally preoperatively and $78 \%$ walking normally postoperatively. Complications of this study included 4 retears and 5 wound complications. Domb et al. ${ }^{14}$ performed a cohort study of 15 patients undergoing endoscopic repair with $100 \%$ patient follow-up at 2 years, which found improvement in all 4 hip-specific scores used to assess clinical outcomes (modified Harris Hip Score, nonarthritic hip score, the Hip Outcome Score-Activities of Daily Living, and the Hip Outcome Score-Sport-Specific subscale), with an average improvement of more than 30 points for all scores. ${ }^{14}$ Satisfaction with the surgery results was reported to

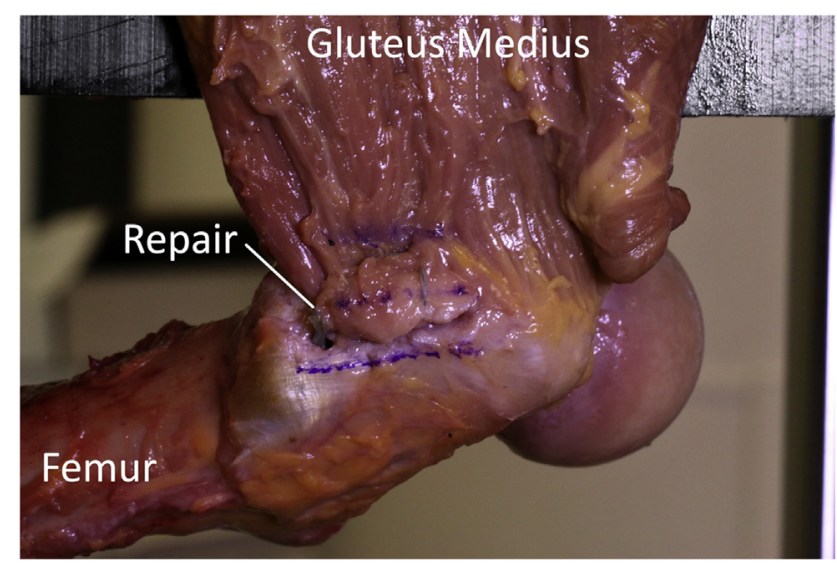

Fig 5. Marker lines at the tendon-bone insertion site and 1 and $2 \mathrm{~cm}$ proximally, which were used to evaluate gap formation during loading. 
Table 1. Biomechanical Summary of Gap Formation Data at the Repair Site of Open and Endoscopic Repair Specimens

\begin{tabular}{lcccccc}
\hline & Ultimate Load, $\mathrm{N}$ & Strain, $\mathrm{mm} / \mathrm{mm}$ & 1st Cycle Gap, mm & 10th Cycle Gap, mm & 150th Cycle Gap, mm & Failure Gap, mm \\
\hline Open & $152.1(68.6)$ & $0.15(0.08)$ & $0.84(0.44)$ & $1.43(0.79)$ & $1.68(0.84)$ & $2.62(1.31)$ \\
Scope & $161.1(72.0)$ & $0.27(0.18)$ & $0.70(0.52)$ & $1.61(0.88)$ & $2.49(1.20)$ & $4.07(2.09)$ \\
$P$ value & .86 & .12 & .63 & .69 & .13 & .15 \\
\hline
\end{tabular}

NOTE. Data are reported as mean (standard deviation). $P$ values are calculated from 2 -tailed paired $t$ tests.

be from good to excellent (7-10 of 10) in 14 of 15 patients. ${ }^{14}$

A systematic review of the literature revealed both endoscopic and open techniques showed significant differences in preoperative and postoperative outcome scores, abduction power, and pain reduction. ${ }^{4}$ The main difference in outcomes in the studies related to the lower complication rates seen with endoscopic surgery. Particularly, open procedures showed a greater retear rate (10 of 128 in the open group compared with 0 in the endoscopic group) in one study. ${ }^{4}$ Open procedures additionally appeared to have a greater incidence of wound complications, specifically infection and hematoma (5 of 128 open vs 0 endoscopic). ${ }^{4}$ Complications seen with the open technique in addition to the ability to arthroscopically visualize the hip joint during the same procedure, provided surgeons with the impetus to pursue and evaluate endoscopic repair. Despite the potential advantages of the endoscopic technique, no study has compared biomechanical fixation strength with open repairs.

Biomechanical studies of shoulders, knees, ankles, and elbows have helped elucidate the failure modes and strengths of repair in cadaveric models. Gluteus medius tears and surgical repair techniques are thought to be analogous to those of the rotator cuff. ${ }^{3,15,16}$ In cadaveric shoulder studies, single-row repairs have performed similarly to double-row repairs in regard to biomechanical fixation. ${ }^{17}$ Failure of these constructs tends to occur at the suture-tendon interface, which is also seen clinically. Double-row techniques have shown greater maximum load to failure with smaller gap formation compared with single-row techniques. ${ }^{18}$ One biomechanical gluteus medius study, from which the loading protocol from this study was modeled, compared 2 open double-row repair techniques (one with massive cuff stitches and the other with knotless lateral anchors) and revealed no difference in cyclic loading outcomes between the two. ${ }^{8}$ It also showed the post-yield extension of the massive cuff stitches was significantly greater than that of the knotless lateral anchors, which the authors concluded was strongly correlated to bone mineral density. ${ }^{8}$

The current biomechanical evaluation of gluteus medius repairs fell short of its goal to quantify the repair strength and elucidate repair failure modes. It revealed, however, both open and endoscopic repairs were stronger than the muscle-bone origin interface, and the authors believe this to be valuable information from which to move forward. It is also crucial to note the larger increase in gap formation between the 1st and 10th cycles compared with the 10th and 150th cycles, indicating the majority of gap formation occurs early in a loading cycle in both open and endoscopic repairs with no significant difference between groups.

\section{Limitations}

Several limiting factors exist in this study. First is the mode of failure of the cadaveric specimens. The custom jig designed for this study enabled the mechanical test frame to hold the specimen and perform cyclic and ramp to failure loading; however, by failing at the origin of the muscle-bone interface, we were unable to quantify the strength or determine the mode of repair failure. In our study, the muscle was left on the ilium in an attempt to preserve the gluteus medius anatomy from origin to insertion, thereby simulating a naturallyoccurring loading environment (aside from the isolation of the gluteus medius). Previous studies have separated the muscle from the ilium and frozen the tissue to create an artificially rigid construct to grip for mechanical testing purposes. Our choice to preserve the overall anatomy and not alter the material properties of structures between the ilium and femur resulted in a lack of failure data. In addition, our biomechanical setup positioned the femur in adduction, whereas a

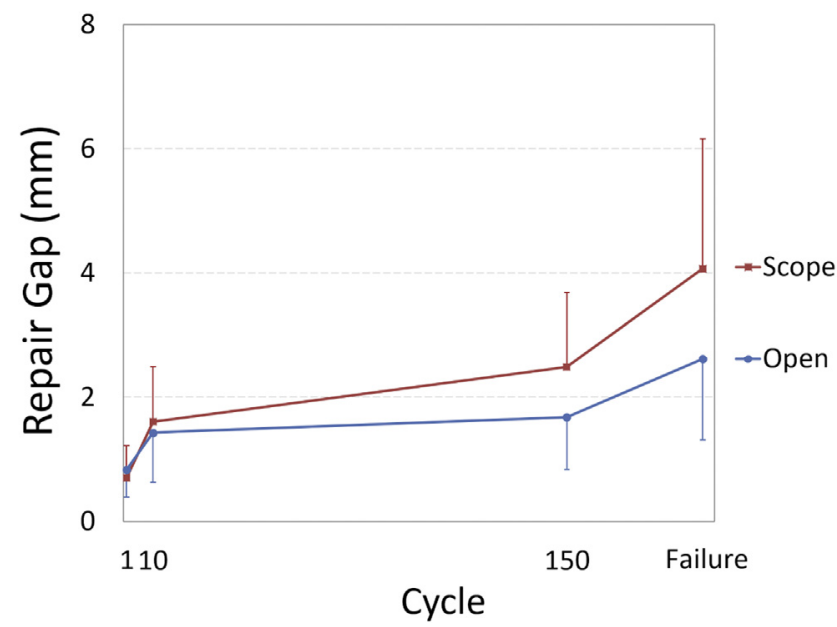

Fig 6. Graph depicting the amount of gap formation between cycles 1-10 and between cycles 10-150. Gap formation increased more during the first 10 cycles than during the subsequent 140 cycles. 
more neutral position may have been more clinically relevant. A further limitation is that the repair groups were tested in a cadaveric model. As such, healing and rehabilitation effects after repair cannot be measured. Clinical outcomes, similarly, are unable to be obtained in a cadaveric model. In addition, we procured cadavers with a maximum age of 50 years for this study in an effort to ensure relatively high bone quality for anchor insertion; however, by doing so, the relatively high tissue quality present in our study does not mimic the poor-quality tissue often seen clinically with complete, retracted gluteus medius tears. Finally, the sample size of 6 pairs of hips may have resulted in the study being underpowered to find significant differences between groups.

\section{Conclusions}

This study shows that both open and endoscopic gluteus medius repairs are stronger than the muscle-bone interface in a cadaveric model and loaded biomechanically in tension between the ilium origin and femoral insertion. Further, endoscopic technique is able to replicate open, knotless gluteus medius repair technique in terms of gap formation in physiologic (i.e., subfailure) cyclic loading.

\section{Acknowledgments}

The authors thank Dr. Alex Brown, Chandler Harris, and Dr. Glenn Fleisig for assistance in conducting these experiments.

\section{References}

1. Pierce TP, Issa K, Kurowicki J, Festa A, McInerney VK, Scillia AJ. Abductor tendon tears of the hip. JBJS Rev 2018;6. doi:10.2106/JBJS.RVW.17.00076.

2. Byrd JWT. Gluteus medius repair with double-row fixation. Arthrosc Tech 2013;2:E247-E250. doi:10.1016/J. EATS.2013.02.015.

3. Bunker TD, Esler CNA, Leach WJ. Rotator-cuff tear of the hip. J Bone Joint Surg Br 1997;79:618-620. doi:10.1302/ 0301-620X.79B4.7033.

4. Chandrasekaran S, Lodhia P, Gui C, Vemula SP, Martin TJ, Domb BG. Outcomes of open versus endoscopic repair of abductor muscle tears of the hip: A systematic review. Arthroscopy 2015;31:2057-2067.e2. doi: 10.1016/j.arthro.2015.03.042.

5. Perets I, Mansor Y, Yuen LC, Chen AW, Chaharbakhshi EO, Domb BG. Endoscopic gluteus medius repair with concomitant arthroscopy for labral tears: A case series with minimum 5-year outcomes. Arthroscopy 2017;33:2159-2167. doi:10.1016/j.arthro.2017.06.032.

6. Byrd JWT. Peritrochanteric access and gluteus medius repair. Arthrosc Tech 2013;2:E243-E246. doi:10.1016/j. eats.2013.02.014.

7. Chandrasekaran S, Gui C, Hutchinson MR, Lodhia P, Suarez-Ahedo C, Domb BG. Outcomes of endoscopic gluteus medius repair: Study of thirty-four patients with minimum two-year follow-up. J Bone Joint Surg Am 2015;97:1340-1347. doi:10.2106/JBJS.N.01229.

8. Dishkin-Paset JG, Salata MJ, Gross CE, et al. A biomechanical comparison of repair techniques for complete gluteus medius tears. Arthroscopy 2012;28: 1410-1416. doi:10.1016/j.arthro.2012.03.002.

9. Voos JE, Shindle MK, Pruett A, Asnis PD, Kelly BT. Endoscopic repair of gluteus medius tendon tears of the hip. Am J Sports Med 2009;37:743-747. doi:10.1177/ 0363546508328412.

10. Bird PA, Oakley SP, Shnier R, Kirkham BW. Prospective evaluation of magnetic resonance imaging and physical examination findings in patients with greater trochanteric pain syndrome. Arthritis Rheum 2001;44:2138-2145. doi: 10.1002/1529-0131(200109)44:9<2138::AID-ART367>3.0. CO;2-M.

11. Kingzett-Taylor A, Tirman PFJ, Feller J, et al. Tendinosis and tears of gluteus medius and minimus muscles as a cause of hip pain: MR imaging findings. Am J Roentgenol 1999;173:1123-1126. doi:10.2214/ajr.173.4.10511191.

12. Chandrasekaran S, Pavan Vemula S, et al. Clinical features that predict the need for operative intervention in gluteus medius tears. Orthop J Sport Med 2015;3:1-5. doi: 10.1177/2325967115571079.

13. Walsh MJ, Walton JR, Walsh NA. Surgical repair of the gluteal tendons. A report of 72 cases. J Arthroplasty 2011;26:1514-1519. doi:10.1016/j.arth.2011.03.004.

14. Domb BG, Botser I, Giordano BD. Outcomes of endoscopic gluteus medius repair with minimum 2-year follow-up. Am J Sports Med 2013;41:988-997. doi:10. $1177 / 0363546513481575$.

15. Domb BG, Carreira DS. Endoscopic repair of full-thickness gluteus medius tears. Arthrosc Tech 2013;2:e77-e81.

16. Hartigan DE, Perets I, Ho SW, Walsh JP, Yuen LC, Domb BG. Endoscopic repair of partial-thickness undersurface tears of the abductor tendon: Clinical outcomes with minimum 2-year follow-up. Arthroscopy 2018;34: 1193-1199. doi:10.1016/j.arthro.2017.10.022.

17. MacGillivray JD, Ma CB. An arthroscopic stitch for massive rotator cuff tears: The mac stitch. Arthroscopy 2004;20:669-671. doi:10.1016/j.arthro.2004.04.065.

18. Lorbach O, Bachelier F, Vees J, Kohn D, Pape D. Cyclic loading of rotator cuff reconstructions: Single-row repair with modified suture configurations versus double-row repair. Am J Sports Med 2008;36:1504-1510. doi:10.1177/ 0363546508314424. 